\title{
RAMSEY NUMBERS FOR TREES
}

\author{
ZHI-HONG SUN
}

(Received 24 September 2011)

\begin{abstract}
For $n \geq 5$, let $T_{n}^{\prime}$ denote the unique tree on $n$ vertices with $\Delta\left(T_{n}^{\prime}\right)=n-2$, and let $T_{n}^{*}=(V, E)$ be the tree on $n$ vertices with $V=\left\{v_{0}, v_{1}, \ldots, v_{n-1}\right\}$ and $E=\left\{v_{0} v_{1}, \ldots, v_{0} v_{n-3}, v_{n-3} v_{n-2}, v_{n-2} v_{n-1}\right\}$. In this paper, we evaluate the Ramsey numbers $r\left(G_{m}, T_{n}^{\prime}\right)$ and $r\left(G_{m}, T_{n}^{*}\right)$, where $G_{m}$ is a connected graph of order $m$. As examples, for $n \geq 8$ we have $r\left(T_{n}^{\prime}, T_{n}^{*}\right)=r\left(T_{n}^{*}, T_{n}^{*}\right)=2 n-5$, for $n>m \geq 7$ we have $r\left(K_{1, m-1}, T_{n}^{*}\right)=m+n-3$ or $m+n-4$ according to whether $m-1 \mid n-3$ or $m-1 \nmid n-3$, and for $m \geq 7$ and $n \geq(m-3)^{2}+2$ we have $r\left(T_{m}^{*}, T_{n}^{*}\right)=m+n-3$ or $m+n-4$ according to whether $m-1 \mid n-3$ or $m-1 \nmid n-3$.
\end{abstract}

2010 Mathematics subject classification: primary 05C35; secondary 05 C05.

Keywords and phrases: Ramsey number, tree, Turán's problem.

\section{Introduction}

In this paper, all graphs are simple graphs. For a graph $G=(V(G), E(G))$ let $e(G)=$ $|E(G)|$ be the number of edges in $G$ and let $\Delta(G)$ be the maximal degree of $G$. For a forbidden graph $L$, let $\operatorname{ex}(p ; L)$ denote the maximal number of edges in a graph of order $p$ not containing $L$ as a subgraph. The corresponding Turán problem is to evaluate $\operatorname{ex}(p ; L)$.

Let $\mathbb{N}$ be the set of positive integers, and let $p, n \in \mathbb{N}$ with $p \geq n \geq 3$. For a given tree $T_{n}$ on $n$ vertices, it is difficult to determine the value of $\operatorname{ex}\left(p ; T_{n}\right)$. The famous ErdôsSós conjecture asserts that $\operatorname{ex}\left(p ; T_{n}\right) \leq(n-2) p / 2$ for every tree $T_{n}$ on $n$ vertices. For progress on the Erdős-Sós conjecture, see [4, 8, 9, 11]. Write $p=k(n-1)+r$, where $k \in \mathbb{N}$ and $r \in\{0,1, \ldots, n-2\}$. Let $P_{n}$ be the path on $n$ vertices. In [5], Faudree and Schelp showed that

$$
\operatorname{ex}\left(p ; P_{n}\right)=k\left(\begin{array}{c}
n-1 \\
2
\end{array}\right)+\left(\begin{array}{l}
r \\
2
\end{array}\right)=\frac{(n-2) p-r(n-1-r)}{2} .
$$

In the special case $r=0,(1.1)$ is due to Erdős and Gallai [3]. Let $K_{1, n-1}$ denote the unique tree on $n$ vertices with $\Delta\left(K_{1, n-1}\right)=n-1$, and for $n \geq 4$ let $T_{n}^{\prime}$ denote the unique

The author is supported by the National Natural Sciences Foundation of China (grant no. 10971078).

(c) 2012 Australian Mathematical Publishing Association Inc. 0004-9727/2012 \$16.00 
tree on $n$ vertices with $\Delta\left(T_{n}^{\prime}\right)=n-2$. In [10], the author and Lin-Lin Wang obtained exact values for $\operatorname{ex}\left(p ; K_{1, n-1}\right)$ and $\operatorname{ex}\left(p ; T_{n}^{\prime}\right)$; see Lemmas 2.4 and 2.5.

For $n \geq 5$ let $T_{n}^{*}=(V, E)$ be the tree on $n$ vertices with $V=\left\{v_{0}, v_{1}, \ldots, v_{n-1}\right\}$ and $E=\left\{v_{0} v_{1}, \ldots, v_{0} v_{n-3}, v_{n-3} v_{n-2}, v_{n-2} v_{n-1}\right\}$. In [10], we also determined the value of $\operatorname{ex}\left(p ; T_{n}^{*}\right)$; see Lemmas 2.6-2.8.

As usual, $\bar{G}$ denotes the complement of a graph $G$. Let $G_{1}$ and $G_{2}$ be two graphs. The Ramsey number $r\left(G_{1}, G_{2}\right)$ is the smallest positive integer $n$ such that, for every graph $G$ with $n$ vertices, either $G$ contains a copy of $G_{1}$ or else $\bar{G}$ contains a copy of $G_{2}$.

Let $n \in \mathbb{N}$ with $n \geq 6$. If the Erdős-Sós conjecture is true, it is known that $r\left(T_{n}, T_{n}\right) \leq$ $2 n-2$ (see [8]). Let $m, n \in \mathbb{N}$. In 1973, Burr and Roberts [2] showed that, for $m, n \geq 3$,

$$
r\left(K_{1, m-1}, K_{1, n-1}\right)= \begin{cases}m+n-3 & \text { if } 2 \nmid m n, \\ m+n-2 & \text { if } 2 \mid m n .\end{cases}
$$

In 1995, Guo and Volkmann [6] proved that, for $n \geq m \geq 5$,

$$
r\left(T_{m}^{\prime}, T_{n}^{\prime}\right)= \begin{cases}m+n-3 & \text { if } m-1 \mid n-3 \\ m+n-5 & \text { if } m=n \equiv 0(\bmod 2) \\ m+n-4 & \text { otherwise }\end{cases}
$$

and, for $n>m \geq 4$,

$$
r\left(K_{1, m-1}, T_{n}^{\prime}\right)= \begin{cases}m+n-3 & \text { if } 2 \mid m(n-1), \\ m+n-4 & \text { if } 2 \nmid m(n-1) .\end{cases}
$$

Let $m, n \in \mathbb{N}$ with $n \geq m \geq 6$. In this paper, we evaluate the Ramsey number $r\left(T_{m}, T_{n}^{*}\right)$ for $T_{m} \in\left\{P_{m}, K_{1, m-1}, T_{m}^{\prime}, T_{m}^{*}\right\}$. As examples, for $n \geq 8$,

$$
r\left(P_{n}, T_{n}^{*}\right)=r\left(T_{n}^{*}, T_{n}^{*}\right)=2 n-5
$$

for $n>m \geq 7$,

$$
r\left(K_{1, m-1}, T_{n}^{*}\right)= \begin{cases}m+n-3 & \text { if } m-1 \mid n-3, \\ m+n-4 & \text { if } m-1 \nmid n-3 ;\end{cases}
$$

and, for $m \geq 7$ and $n \geq(m-3)^{2}+2$,

$$
r\left(P_{m}, T_{n}^{*}\right)=r\left(T_{m}^{\prime}, T_{n}^{*}\right)=r\left(T_{m}^{*}, T_{n}^{*}\right)= \begin{cases}m+n-3 & \text { if } m-1 \mid n-3, \\ m+n-4 & \text { if } m-1 \nmid n-3 .\end{cases}
$$

In addition to the above notation, throughout the paper we also use the following notation: $\lfloor x\rfloor$ is the greatest integer not exceeding $x, K_{n}$ is the complete graph on $n$ vertices, $K_{m, n}$ is the complete bipartite graph with $m$ and $n$ vertices in the 
bipartition, $d_{G}(v)$ is the degree of the vertex $v$ in a given graph $G$, and $d(u, v)$ is the distance between the two vertices $u$ and $v$ in a graph.

\section{Basic lemmas}

Lemma 2.1. Let $G_{1}$ and $G_{2}$ be two graphs. Suppose that $p \in \mathbb{N}, p \geq \max \left\{\left|V\left(G_{1}\right)\right|\right.$, $\left.\left|V\left(G_{2}\right)\right|\right\}$ and $\operatorname{ex}\left(p ; G_{1}\right)+\operatorname{ex}\left(p ; G_{2}\right)<\left(\begin{array}{l}p \\ 2\end{array}\right)$. Then $r\left(G_{1}, G_{2}\right) \leq p$.

Proof. Let $G$ be a graph of order $p$. If $e(G) \leq \operatorname{ex}\left(p ; G_{1}\right)$ and $e(\bar{G}) \leq \operatorname{ex}\left(p ; G_{2}\right)$, then

$$
\operatorname{ex}\left(p ; G_{1}\right)+\operatorname{ex}\left(p ; G_{2}\right) \geq e(G)+e(\bar{G})=\left(\begin{array}{l}
p \\
2
\end{array}\right)
$$

This contradicts the assumption. Hence, either $e(G)>\operatorname{ex}\left(p ; G_{1}\right)$ or $e(\bar{G})>\operatorname{ex}\left(p ; G_{2}\right)$. Therefore, $G$ contains a copy of $G_{1}$ or $\bar{G}$ contains a copy of $G_{2}$. This shows that $r\left(G_{1}, G_{2}\right) \leq|V(G)|=p$. So the lemma is proved.

Lemma 2.2. Let $k, p \in \mathbb{N}$ with $p \geq k+1$. Then there exists a $k$-regular graph of order $p$ if and only if $2 \mid \mathrm{kp}$.

This is a known result; see, for example, [10, Corollary 2.1].

Lemma 2.3. Let $G_{1}$ and $G_{2}$ be two graphs with $\Delta\left(G_{1}\right)=d_{1} \geq 2$ and $\Delta\left(G_{2}\right)=d_{2} \geq 2$. Then the following results hold.

(i) $\quad r\left(G_{1}, G_{2}\right) \geq d_{1}+d_{2}-\left(1-(-1)^{\left(d_{1}-1\right)\left(d_{2}-1\right)}\right) / 2$.

(ii) Suppose that $G_{1}$ is a connected graph of order $m$ and $d_{1}<d_{2} \leq m$. Then $r\left(G_{1}, G_{2}\right) \geq 2 d_{2}-1 \geq d_{1}+d_{2}$.

(iii) Suppose that $G_{1}$ is a connected graph of order $m$ and $d_{2}>m$. Then $r\left(G_{1}, G_{2}\right) \geq$ $d_{1}+d_{2}$ if one of the following conditions holds:

(1) $2 \mid d_{1}+d_{2}-m$;

(2) $d_{1} \neq m-1$;

(3) $G_{2}$ has two vertices $u$ and $v$ such that $d(v)=\Delta\left(G_{2}\right)$ and $d(u, v)=3$.

Proof. We first consider (i). If $2 \mid\left(d_{1}-1\right)\left(d_{2}-1\right)$, then $2 \mid\left(d_{1}-1\right)\left(d_{1}+d_{2}-1\right)$. Since $d_{1}-1 \geq 1$, by Lemma 2.2 we may construct a $\left(d_{1}-1\right)$-regular graph $G$ of order $d_{1}+$ $d_{2}-1$. Since $\Delta(G)=d_{1}-1$ and $\Delta(\bar{G})=d_{2}-1, G$ does not contain $G_{1}$ as a subgraph and $\bar{G}$ does not contain $G_{2}$ as a subgraph. Hence $r\left(G_{1}, G_{2}\right) \geq 1+|V(G)|=d_{1}+d_{2}$. Now we assume that $2 \nmid\left(d_{1}-1\right)\left(d_{2}-1\right)$. Then $2\left|d_{1}, 2\right| d_{2}$ and so $2 \mid d_{1}+d_{2}-2$. By Lemma 2.2, we may construct a $\left(d_{1}-1\right)$-regular graph $G$ of order $d_{1}+d_{2}-2$. Since $\Delta(G)=d_{1}-1$ and $\Delta(\bar{G})=d_{2}-2, G$ does not contain $G_{1}$ as a subgraph and $\bar{G}$ does not contain $G_{2}$ as a subgraph. Hence $r\left(G_{1}, G_{2}\right) \geq 1+|V(G)|=d_{1}+d_{2}-1$. This proves (i). 
Next we consider (ii). Suppose that $G_{1}$ is a connected graph of order $m$ and $d_{1}<d_{2} \leq m$. Since $K_{d_{2}-1} \cup K_{d_{2}-1}$ does not contain any copies of $G_{1}$, and its complement $K_{d_{2}-1, d_{2}-1}$ does not contain any copies of $G_{2}$, we see that

$$
r\left(G_{1}, G_{2}\right) \geq 1+2\left(d_{2}-1\right)=2 d_{2}-1 \geq d_{1}+d_{2} .
$$

This proves (ii).

Finally, we consider (iii). Suppose that $G_{1}$ is a connected graph of order $m$ and $d_{2}>m$. By Lemma 2.2, we may construct a graph

$$
G= \begin{cases}K_{m-1} \cup H_{1} & \text { if } 2 \mid d_{1}+d_{2}-m, \\ K_{m-2} \cup H_{2} & \text { if } 2 \nmid d_{1}+d_{2}-m,\end{cases}
$$

where $H_{1}$ is a $\left(d_{1}-1\right)$-regular graph of order $d_{1}+d_{2}-m$ and $H_{2}$ is a $\left(d_{1}-1\right)$-regular graph of order $d_{1}+d_{2}-m+1$. It is easily seen that $G$ does not contain any copies of $G_{1}$ and

$$
\Delta(\bar{G})= \begin{cases}d_{2}-1 & \text { if } 2 \mid d_{1}+d_{2}-m \text { or } d_{1} \neq m-1, \\ d_{2} & \text { if } 2 \nmid d_{1}+d_{2}-m \text { and } d_{1}=m-1 .\end{cases}
$$

If $2 \mid d_{1}+d_{2}-m$ or $d_{1} \neq m-1$, then $\bar{G}$ does not contain any copies of $G_{2}$ and so $r\left(G_{1}, G_{2}\right) \geq 1+|V(G)|=d_{1}+d_{2}$. Now assume that $2 \nmid d_{1}+d_{2}-m$ and $d_{1}=$ $m-1$. For $v_{0} \in V\left(H_{2}\right), d_{\bar{G}}\left(v_{0}\right)=d_{2}-1$. Suppose that $v_{1}, \ldots, v_{m-2} \in V(G)$ and $v_{1}, \ldots, v_{m-2}$ induce a copy of $K_{m-2}$. Then $\left\{v_{1}, \ldots, v_{m-2}\right\}$ is an independent set in $\bar{G}$ and $d_{\bar{G}}\left(v_{i}\right)=d_{2}$ for $i=1,2, \ldots, m-2$. If $G_{2}$ has two vertices $u$ and $v$ such that $d(v)=\Delta\left(G_{2}\right)$ and $d(u, v)=3$, we see that $G$ does not contain any copies of $G_{2}$ and so $r\left(G_{1}, G_{2}\right) \geq 1+|V(G)|=d_{1}+d_{2}$. This proves (iii) and the lemma is proved.

Lemma 2.4 [10, Theorem 2.1]. Let $p, n \in \mathbb{N}$ with $p \geq n-1 \geq 1$. Then $\operatorname{ex}\left(p ; K_{1, n-1}\right)=$ $\lfloor(n-2) p / 2\rfloor$.

Lemma 2.5 [10, Theorem 3.1]. Let $p, n \in \mathbb{N}$ with $p \geq n \geq 5$. Let $r \in\{0,1, \ldots, n-2\}$ be given by $p \equiv r(\bmod n-1)$. Then

$$
\operatorname{ex}\left(p ; T_{n}^{\prime}\right)= \begin{cases}\left\lfloor\frac{(n-2)(p-1)-r-1}{2}\right\rfloor & \text { if } n \geq 7 \text { and } 2 \leq r \leq n-4, \\ \frac{(n-2) p-r(n-1-r)}{2} & \text { otherwise. }\end{cases}
$$

Lemma 2.6 [10, Theorems 4.1-4.3]. Let $p, n \in \mathbb{N}$ with $p \geq n \geq 6$, and let $p=k(n-1)+$ $r$ with $k \in \mathbb{N}$ and $r \in\{0,1, n-5, n-4, n-3, n-2\}$. Then

$$
\operatorname{ex}\left(p ; T_{n}^{*}\right)= \begin{cases}\frac{(n-2)(p-2)}{2}+1 & \text { if } n>6 \text { and } r=n-5, \\ \frac{(n-2) p-r(n-1-r)}{2} & \text { otherwise. }\end{cases}
$$


Lemma 2.7 [10, Theorem 4.4]. Let $p, n \in \mathbb{N}, p \geq n \geq 11, r \in\{2,3, \ldots, n-6\}$ and $p \equiv r(\bmod n-1)$. Let $t \in\{0,1, \ldots, r+1\}$ be given by $n-3 \equiv t(\bmod r+2)$. Then

$$
\operatorname{ex}\left(p ; T_{n}^{*}\right)= \begin{cases}\left\lfloor\frac{(n-2)(p-1)-2 r-t-3}{2}\right\rfloor & \text { if } r \geq 4 \text { and } 2 \leq t \leq r-1, \\ \frac{(n-2)(p-1)-t(r+2-t)-r-1}{2} & \text { otherwise. }\end{cases}
$$

Lemma 2.8 [10, Theorem 4.5]. Let $p, n \in \mathbb{N}$ with $6 \leq n \leq 10$ and $p \geq n$, and let $r \in\{0,1, \ldots, n-2\}$ be given by $p \equiv r(\bmod n-1)$.

(i) If $n=6,7$, then $\operatorname{ex}\left(p ; T_{n}^{*}\right)=((n-2) p-r(n-1-r)) / 2$.

(ii) If $n=8,9$, then

$$
\operatorname{ex}\left(p ; T_{n}^{*}\right)= \begin{cases}\frac{(n-2) p-r(n-1-r)}{2} & \text { if } r \neq n-5, \\ \frac{(n-2)(p-2)}{2}+1 & \text { if } r=n-5 .\end{cases}
$$

(iii) If $n=10$, then

$$
\operatorname{ex}\left(p ; T_{n}^{*}\right)= \begin{cases}4 p-\frac{r(9-r)}{2} & \text { if } r \neq 4,5 \\ 4 p-7 & \text { if } r=5 \\ 4 p-9 & \text { if } r=4\end{cases}
$$

Lemma 2.9. Let $p, m \in \mathbb{N}$ with $p \geq m \geq 5$, and $T_{m} \in\left\{P_{m}, K_{1, m-1}, T_{m}^{\prime}, T_{m}^{*}\right\}$. Then $\operatorname{ex}\left(p ; T_{m}\right) \leq(m-2) p / 2$. Moreover, if $m-1 \nmid p$ and $T_{m} \in\left\{P_{m}, T_{m}^{\prime}, T_{m}^{*}\right\}$, then $\operatorname{ex}\left(p ; T_{m}\right) \leq(m-2)(p-1) / 2$.

Proof. This is immediate from (1.1) and Lemmas 2.4-2.8.

Lemma 2.10. Let $m, n \in \mathbb{N}$ with $m, n \geq 5$. Let $G_{m}$ be a connected graph on $m$ vertices. If $m+n-5=(m-1) x+(m-2) y$ for some nonnegative integers $x$ and $y$, then $r\left(G_{m}, T_{n}\right) \geq m+n-4$ for $T_{n} \in\left\{K_{1, n-1}, T_{n}^{\prime}, T_{n}^{*}\right\}$.

Proof. Let $G=x K_{m-1} \cup y K_{m-2}$. Then $|V(G)|=m+n-5, \Delta(G) \leq m-1$ and $\Delta(\bar{G}) \leq$ $n-3$. Clearly, $G$ does not contain $G_{m}$ as a subgraph, and $\bar{G}$ does not contain $T_{n}$ as a subgraph. So the result is true.

Lemma 2.11 [7, Theorem 8.3, pp. 11-12]. Let $a, b, n \in \mathbb{N}$. If a is coprime to $b$ and $n \geq(a-1)(b-1)$, then there are two nonnegative integers $x$ and $y$ such that $n=a x+b y$.

Conjecture 2.12. Let $p, n \in \mathbb{N}, p \geq n \geq 5, p=k(n-1)+r, k \in \mathbb{N}$ and $r \in\{0,1, \ldots$, $n-2\}$. Let $T_{n} \neq K_{1, n-1}, T_{n}^{\prime}$ be a tree on $n$ vertices. Then $\operatorname{ex}\left(p ; T_{n}\right) \leq \operatorname{ex}\left(p ; T_{n}^{*}\right)$. Hence: 
(i) if $r \in\{0,1, n-4, n-3, n-2\}$, then

$$
\operatorname{ex}\left(p ; T_{n}\right)=\frac{(n-2) p-r(n-1-r)}{2} ;
$$

(ii) if $2 \leq r \leq n-5$, then

$$
\operatorname{ex}\left(p ; T_{n}\right) \leq \frac{(n-2)(p-1)-r-1}{2}
$$

We note that

$$
\operatorname{ex}\left(p ; T_{n}\right) \geq e\left(k K_{n-1} \cup K_{r}\right)=\frac{(n-2) p-r(n-1-r)}{2}=\operatorname{ex}\left(p ; P_{n}\right) .
$$

Definition 2.13. For $n \geq 5$ let $T_{n}$ be a tree on $n$ vertices. View $T_{n}$ as a bipartite graph with $s_{1}$ and $s_{2}$ vertices in the bipartition. Define $\alpha_{2}\left(T_{n}\right)=\max \left\{s_{1}, s_{2}\right\}$.

Conjecture 2.14. Let $p, n \in \mathbb{N}$ with $p \geq n \geq 5$. Let $T_{n}^{(1)}$ and $T_{n}^{(2)}$ be two trees on $n$ vertices. If $\alpha_{2}\left(T_{n}^{(1)}\right)<\alpha_{2}\left(T_{n}^{(2)}\right)$, then $\operatorname{ex}\left(p ; T_{n}^{(1)}\right) \leq \operatorname{ex}\left(p ; T_{n}^{(2)}\right)$.

\section{The Ramsey number $r\left(G_{n}, T_{n}^{*}\right)$}

Lemma 3.1. Let $n \in \mathbb{N}, n \geq 6$, and let $G_{n}$ be a connected graph on $n$ vertices such that $\operatorname{ex}\left(2 n-5 ; G_{n}\right)<n^{2}-5 n+4$. Then $r\left(G_{n}, T_{n}^{*}\right)=2 n-5$.

Proof. As $2 K_{n-3}$ does not contain any copies of $G_{n}$ and $\overline{2 K_{n-3}}=K_{n-3, n-3}$ does not contain any copies of $T_{n}^{*}$, we see that $r\left(G_{n}, T_{n}^{*}\right)>2(n-3)$. By Lemma 2.6,

$$
\operatorname{ex}\left(2 n-5 ; T_{n}^{*}\right)=\frac{(n-2)(2 n-5)-3(n-4)}{2}=n^{2}-6 n+11
$$

Thus,

$$
\begin{aligned}
\operatorname{ex}\left(2 n-5 ; G_{n}\right)+\operatorname{ex}\left(2 n-5 ; T_{n}^{*}\right) & <n^{2}-5 n+4+n^{2}-6 n+11 \\
& =2 n^{2}-11 n+15=\left(\begin{array}{c}
2 n-5 \\
2
\end{array}\right)
\end{aligned}
$$

Appealing to Lemma 2.1, we obtain $r\left(G_{n}, T_{n}^{*}\right) \leq 2 n-5$. So $r\left(G_{n}, T_{n}^{*}\right)=2 n-5$ as asserted.

Theorem 3.2. Let $n \in \mathbb{N}$ with $n \geq 8$. Then

$$
r\left(P_{n}, T_{n}^{*}\right)=r\left(T_{n}^{\prime}, T_{n}^{*}\right)=r\left(T_{n}^{*}, T_{n}^{*}\right)=2 n-5 .
$$

Proof. By Lemma 2.6,

$$
\operatorname{ex}\left(2 n-5 ; T_{n}^{*}\right)=\frac{(n-2)(2 n-5)-3(n-4)}{2}=n^{2}-6 n+11<n^{2}-5 n+4
$$


By Lemma 2.5,

$$
\begin{aligned}
\operatorname{ex}\left(2 n-5 ; T_{n}^{\prime}\right) & =\left\lfloor\frac{(n-2)(2 n-6)-(n-4)-1}{2}\right\rfloor=\left\lfloor n^{2}-\frac{11}{2} n+\frac{15}{2}\right\rfloor \\
& \leq n^{2}-\frac{11}{2} n+\frac{15}{2}<n^{2}-5 n+4
\end{aligned}
$$

By (1.1),

$$
\operatorname{ex}\left(2 n-5 ; P_{n}\right)=\left(\begin{array}{c}
n-1 \\
2
\end{array}\right)+\left(\begin{array}{c}
n-4 \\
2
\end{array}\right)=n^{2}-6 n+11<n^{2}-5 n+4
$$

Thus, applying Lemma 3.1, we deduce the result.

Conjecture 3.3. Let $n \in \mathbb{N}, n \geq 8$, and let $T_{n} \neq K_{1, n-1}$ be a tree on $n$ vertices. Then $r\left(T_{n}, T_{n}^{*}\right)=2 n-5$.

Remark 3.4. Let $n \in \mathbb{N}$ with $n \geq 4$. From [6, Theorem 3.1(ii)] we know that $r\left(K_{1, n-1}, T_{n}^{*}\right)=2 n-3$.

\section{The Ramsey number $r\left(G_{m}, T_{n}^{*}\right)$ for $m<n$}

THEOREM 4.1. Let $m, n \in \mathbb{N}, n>m \geq 5$ and $m-1 \mid n-3$. Let $G_{m}$ be a connected graph of order $m$ such that

$$
\operatorname{ex}\left(m+n-3 ; G_{m}\right) \leq \frac{(m-2)(m+n-3)}{2} \quad \text { or } \quad G_{m} \in\left\{P_{m}, K_{1, m-1}, T_{m}^{\prime}, T_{m}^{*}\right\} .
$$

Then $r\left(G_{m}, T_{n}^{*}\right)=m+n-3$.

Proof. By Lemma 2.9 we may assume that ex $\left(m+n-3 ; G_{m}\right) \leq(m-2)(m+n-3) / 2$. Suppose that $n-3=k(m-1)$. Clearly $(k+1) K_{m-1}$ does not contain $G_{m}$ as a subgraph and $\overline{(k+1) K_{m-1}}$ does not contain $T_{n}^{*}$ as a subgraph. Thus,

$$
r\left(G_{m}, T_{n}^{*}\right)>(k+1)(m-1)=m+n-4 .
$$

Since $1 \leq m-4 \leq n-6$, using Lemma 2.9 we see that

$$
\operatorname{ex}\left(m+n-3 ; T_{n}^{*}\right) \leq \frac{(n-2)(m+n-4)}{2} .
$$

Thus,

$$
\begin{aligned}
& \operatorname{ex}\left(m+n-3 ; G_{m}\right)+\operatorname{ex}\left(m+n-3 ; T_{n}^{*}\right) \\
& \quad \leq \frac{(m-2)(m+n-3)}{2}+\frac{(n-2)(m+n-4)}{2} \\
& \quad<\frac{(m-2+n-2)(m+n-3)}{2}=\left(\begin{array}{c}
m+n-3 \\
2
\end{array}\right) .
\end{aligned}
$$

Hence, by Lemma 2.1, $r\left(G_{m}, T_{n}^{*}\right) \leq m+n-3$, and the result follows. 
Lemma 4.2. Let $m, n \in \mathbb{N}, n>m \geq 7$ and $m-1 \nmid n-3$. Let $G_{m}$ be a connected graph of order $m$ such that

$$
\operatorname{ex}\left(m+n-4 ; G_{m}\right) \leq \frac{(m-2)(m+n-4)}{2} \quad \text { or } \quad G_{m} \in\left\{P_{m}, K_{1, m-1}, T_{m}^{\prime}, T_{m}^{*}\right\}
$$

Then $r\left(G_{m}, T_{n}^{*}\right) \leq m+n-4$.

Proof. By Lemma 2.9, we may assume that

$$
\operatorname{ex}\left(m+n-4 ; G_{m}\right) \leq(m-2)(m+n-4) / 2 .
$$

As $m+n-4=n-1+m-3$ and $m-1 \nmid n-3$, we see that $2 \leq m-3 \leq n-4$ and $m-3 \neq n-5$. Thus, applying Lemmas 2.6-2.8,

$$
\operatorname{ex}\left(m+n-4 ; T_{n}^{*}\right)<\frac{(n-3)(m+n-4)}{2} .
$$

Hence,

$$
\begin{aligned}
& \operatorname{ex}\left(m+n-4 ; G_{m}\right)+\operatorname{ex}\left(m+n-4 ; T_{n}^{*}\right) \\
& \quad<\frac{(m-2)(m+n-4)}{2}+\frac{(n-3)(m+n-4)}{2}=\left(\begin{array}{c}
m+n-4 \\
2
\end{array}\right) .
\end{aligned}
$$

Applying Lemma 2.1, we obtain the result.

Theorem 4.3. Let $m, n \in \mathbb{N}, n>m \geq 7$ and $m-1 \nmid n-3$. Let $G_{m}$ be a connected graph of order $m$ such that

$$
\operatorname{ex}\left(m+n-4 ; G_{m}\right) \leq \frac{(m-2)(m+n-4)}{2} \quad \text { or } \quad G_{m} \in\left\{P_{m}, T_{m}^{\prime}, T_{m}^{*}\right\}
$$

If $m+n-5=(m-1) x+(m-2) y$ for some $x, y \in\{0,1,2, \ldots\}$, then $r\left(G_{m}, T_{n}^{*}\right)=m+$ $n-4$.

Proof. By Lemma 4.2, $r\left(G_{m}, T_{n}^{*}\right) \leq m+n-4$, and by Lemma 2.10, $r\left(G_{m}, T_{n}^{*}\right) \geq$ $m+n-4$. Thus the result follows.

Theorem 4.4. Suppose that $m, n \in \mathbb{N}, \quad n>m \geq 7, \quad n=k(m-1)+b=q(m-2)+a$, $k, q \in \mathbb{N}, a \in\{0,1, \ldots, m-3\}$ and $b \in\{0,1, \ldots, m-2\}-\{3\}$. Let $G_{m}$ be a connected graph of order $m$ such that

$$
\operatorname{ex}\left(m+n-4 ; G_{m}\right) \leq \frac{(m-2)(m+n-4)}{2} \quad \text { or } \quad G_{m} \in\left\{P_{m}, T_{m}^{\prime}, T_{m}^{*}\right\} .
$$

If one of the conditions:

(i) $b \in\{1,2,4\}$;

(ii) $b=0$ and $k \geq 3$; 
(iii) $n \geq(m-3)^{2}+2$;

(iv) $n \geq m^{2}-1-b(m-2)$; or

(v) $a \geq 3$ and $n \geq(a-4)(m-1)+4$

holds, then $r\left(G_{m}, T_{n}^{*}\right)=m+n-4$.

Proof. For $b \in\{1,2,4\}$,

$$
m+n-5= \begin{cases}(k-2)(m-1)+3(m-2) & \text { if } b=1 \\ (k-1)(m-1)+2(m-2) & \text { if } b=2 \\ (k+1)(m-1) & \text { if } b=4\end{cases}
$$

For $b=0$ and $k \geq 3, m+n-5=(k-3)(m-1)+4(m-2)$. For $n \geq(m-3)^{2}+2$, $m+n-5 \geq(m-2)(m-3)$ and so $m+n-5=(m-1) x+(m-2) y$ for some $x, y \in$ $\{0,1,2, \ldots\}$ by Lemma 2.11. For $n \geq m^{2}-1-b(m-2), k \geq m+1-b$ and

$$
m+n-5=(k+b-m-1)(m-1)+(m+3-b)(m-2) .
$$

For $a \geq 3$ and $n \geq(a-4)(m-1)+4, q \geq a-4$ and

$$
m+n-5=(a-3)(m-1)+(q+4-a)(m-2) .
$$

Combining all the above with Theorem 4.3, we obtain the result.

Theorem 4.5. Suppose that $m, n \in \mathbb{N}, n>m \geq 7$ and $m-1 \nmid n-3$. Then

$$
\begin{gathered}
r\left(K_{1, m-1}, T_{n}^{*}\right)=m+n-4, \\
r\left(T_{m}^{\prime}, T_{n}^{*}\right)=m+n-4 \text { or } m+n-5, \\
m+n-6 \leq r\left(T_{m}^{*}, T_{n}^{*}\right) \leq m+n-4 .
\end{gathered}
$$

Proof. From Lemma 4.2,

$$
r\left(T_{m}, T_{n}^{*}\right) \leq m+n-4 \text { for } T_{m} \in\left\{K_{1, m-1}, T_{m}^{\prime}, T_{m}^{*}\right\} .
$$

By Lemma 2.3,

$$
r\left(K_{1, m-1}, T_{n}^{*}\right) \geq m-1+n-3, \quad r\left(T_{m}^{\prime}, T_{n}^{*}\right) \geq m-2+n-3 \quad(n>m+1)
$$

and

$$
r\left(T_{m}^{*}, T_{n}^{*}\right) \geq m-3+n-3 .
$$

By Theorem 4.4, $r\left(T_{m}^{\prime}, T_{n}^{*}\right)=m+n-4$ for $n=1, m+3$. Thus the theorem is proved.

Theorem 4.6. Suppose that $m, n \in \mathbb{N}, \quad n>m \geq 7, \quad n=k(m-1)+b, \quad k \in \mathbb{N}, \quad b \in$ $\{0,1, \ldots, m-2\}, b \neq 3$ and $(m-b) / 2 \leq k \leq m+2-b$. Let $G_{m}$ be a connected graph of order $m$ such that $\operatorname{ex}\left(m+n-4 ; G_{m}\right) \leq \frac{1}{2}(m-2)(m+n-4)$ or $G_{m} \in\left\{P_{m}, T_{m}^{*}\right\}$. Then $r\left(G_{m}, T_{n}^{*}\right)=m+n-4$ or $m+n-5$. 
Proof. By Lemma 4.2 we only need to show that $r\left(G_{m}, T_{n}^{*}\right)>m+n-6$. Set

$$
G=(2 k+b-m) K_{m-2} \cup(m+2-b-k) K_{m-3} .
$$

Then

$$
|V(G)|=(2 k+b-m)(m-2)+(m+2-b-k)(m-3)=m+n-6 .
$$

We also have $\Delta(G) \leq m-2$ and $\Delta(\bar{G}) \leq m+n-6-(m-3)=n-3$. Now it is clear that $G_{m}$ is not a subgraph of $G$ and that $T_{n}^{*}$ is not a subgraph of $\bar{G}$. So $r\left(G_{m}, T_{n}^{*}\right)>$ $|V(G)|$, which completes the proof.

REMARK 4.7. If $p \geq m \geq 6$ and $T_{m}$ is a tree on $m$ vertices with a vertex adjacent to at least $\lfloor(m-1) / 2\rfloor$ vertices of degree one, in [9] Sidorenko proved that $\operatorname{ex}\left(p ; T_{m}\right) \leq$ $(m-2) p / 2$. Thus, $G_{m}$ can be replaced by $T_{m}$ in Lemma 4.2 and Theorems 4.1, 4.3, 4.4 and 4.6 .

\section{The Ramsey number $r\left(G_{m}, T_{n}^{\prime}\right)$ for $m<n$}

Theorem 5.1. Let $m, n \in \mathbb{N}, n>m \geq 6$ and $m-1 \mid n-3$. Suppose that $G_{m}$ is a connected graph of order $m$ satisfying

$$
\operatorname{ex}\left(m+n-3 ; G_{m}\right) \leq \frac{(m-2)(m+n-3)+m+n-4}{2} \quad \text { or } \quad G_{m} \in\left\{T_{m}^{*}, P_{m}\right\} .
$$

Then $r\left(G_{m}, T_{n}^{\prime}\right)=m+n-3$.

Proof. By Lemma 2.9 we may assume that

$$
\operatorname{ex}\left(m+n-3 ; G_{m}\right) \leq(m-2)(m+n-3) / 2+(m+n-4) / 2 .
$$

Suppose that $n-3=k(m-1)$ and $G=(k+1) K_{m-1}$. Then $|V(G)|=m+n-4$ and $\Delta(\bar{G})=n-3$. Clearly, $G_{m}$ is not a subgraph of $G$ and $T_{n}^{\prime}$ is not a subgraph of $\bar{G}$. Thus $r\left(G_{m}, T_{n}^{\prime}\right)>m+n-4$. Since $m-1 \mid n-3, n \geq m+2$ and so $4 \leq m-2 \leq n-4$. Hence, using Lemma 2.5,

$$
\begin{aligned}
\operatorname{ex}\left(m+n-3 ; T_{n}^{\prime}\right) & =\left\lfloor\frac{(n-2)(m+n-4)-(m-1)}{2}\right\rfloor \\
& <\frac{(n-2)(m+n-3)-(m+n-4)}{2} .
\end{aligned}
$$

Therefore

$$
\operatorname{ex}\left(m+n-3 ; G_{m}\right)+\operatorname{ex}\left(m+n-3 ; T_{n}^{\prime}\right)<\left(\begin{array}{c}
m+n-3 \\
2
\end{array}\right) .
$$

Applying Lemma 2.1, we see that $r\left(G_{m}, T_{n}^{\prime}\right) \leq m+n-3$, so the result follows.

Lemma 5.2. Let $m, n \in \mathbb{N}, n>m \geq 6$ and $m-1 \nmid n-3$. Suppose that $G_{m}$ is a connected graph of order $m$ satisfying $\operatorname{ex}\left(m+n-4 ; G_{m}\right)<(m-2)(m+n-4) / 2$ or $G_{m} \in\left\{T_{m}^{*}, P_{m}\right\}$. Then $r\left(G_{m}, T_{n}^{\prime}\right) \leq m+n-4$. 
Proof. Since $m-1 \nmid n-3, m-1 \nmid m+n-4$. Thus, applying Lemma 2.9,

$$
\operatorname{ex}\left(m+n-4 ; T_{m}^{*}\right) \leq(m-2)(m+n-5) / 2
$$

and

$$
\operatorname{ex}\left(m+n-4 ; P_{m}\right) \leq(m-2)(m+n-5) / 2 .
$$

As $n>m, 3 \leq m-3 \leq n-4$. By Lemma 2.5,

$$
\begin{aligned}
\operatorname{ex}\left(m+n-4 ; T_{n}^{\prime}\right) & =\left\lfloor\frac{(n-2)(m+n-5)-(m-2)}{2}\right\rfloor \\
& \leq \frac{(n-2)(m+n-5)-(m-2)}{2} .
\end{aligned}
$$

Thus

$$
\begin{aligned}
\operatorname{ex}\left(m+n-4 ; G_{m}\right)+\operatorname{ex}\left(m+n-4 ; T_{n}^{\prime}\right) & <\frac{(m-2+n-2)(m+n-5)}{2} \\
& =\left(\begin{array}{c}
m+n-4 \\
2
\end{array}\right) .
\end{aligned}
$$

This, together with Lemma 2.1, yields the result.

Theorem 5.3. Let $m, n \in \mathbb{N}, n>m \geq 6$ and $m-1 \nmid n-3$. Then $r\left(T_{m}^{*}, T_{m+1}^{\prime}\right)=2 m-3$ and $r\left(T_{m}^{*}, T_{n}^{\prime}\right)=m+n-4$ or $m+n-5$ for $n \geq m+3$. Suppose that $G_{m}$ is a connected graph of order $m$ satisfying $\operatorname{ex}\left(m+n-4 ; G_{m}\right)<(m-2)(m+n-4) / 2$ or $G_{m} \in\left\{T_{m}^{*}, P_{m}\right\}$. If $m+n-5=(m-1) x+(m-2) y$ for some nonnegative integers $x$ and $y$, then $r\left(G_{m}, T_{n}^{\prime}\right)=m+n-4$.

Proof. By Lemma 2.3,

$$
r\left(T_{m}^{*}, T_{m+1}^{\prime}\right) \geq 2(m-1)-1=2 m-3
$$

and

$$
r\left(T_{m}^{*}, T_{n}^{\prime}\right) \geq m-3+n-2 \text { for } n \geq m+3 .
$$

By Lemma 5.2, $r\left(G_{m}, T_{n}^{\prime}\right) \leq m+n-4$. Thus, $r\left(T_{m}^{*}, T_{m+1}^{\prime}\right)=2 m-3$. Applying Lemma 2.10, we deduce the remaining result.

From Theorem 5.3 and the proof of Theorem 4.4 we deduce the following result.

Theorem 5.4. Suppose that $m, n \in \mathbb{N}, \quad n>m \geq 6, n=k(m-1)+b=q(m-2)+a$, $k, q \in \mathbb{N}, a \in\{0,1, \ldots, m-3\}$ and $b \in\{0,1, \ldots, m-2\}-\{3\}$. Let $G_{m}$ be a connected graph of order $m$ such that $\operatorname{ex}\left(m+n-4 ; G_{m}\right)<(m-2)(m+n-4) / 2$ or $G_{m} \in$ $\left\{P_{m}, T_{m}^{*}\right\}$. If one of the conditions:

(i) $b \in\{1,2,4\}$;

(ii) $b=0$ and $k \geq 3$;

(iii) $n \geq(m-3)^{2}+2$;

(iv) $n \geq m^{2}-1-b(m-2)$; or

(v) $\quad a \geq 3$ and $n \geq(a-4)(m-1)+4$

holds, then $r\left(G_{m}, T_{n}^{\prime}\right)=m+n-4$. 


\section{The Ramsey number $r\left(T_{m}, K_{1, n-1}\right)$ for $m<n$}

The following two propositions are known.

Proposition 6.1 [1]. Let $m, n \in \mathbb{N}$ with $m \geq 3$ and $m-1 \mid n-2$. Let $T_{m}$ be a tree on $m$ vertices. Then $r\left(T_{m}, K_{1, n-1}\right)=m+n-2$.

Proposition 6.2 [6, Theorem 3.1]. Let $m, n \in \mathbb{N}, m \geq 3$ and $n=k(m-1)+b$ with $k \in \mathbb{N}$ and $b \in\{0,1, \ldots, m-2\}-\{2\}$. Let $T_{m} \neq K_{1, m-1}$ be a tree on $m$ vertices. Then $r\left(T_{m}, K_{1, n-1}\right) \leq m+n-3$. Moreover, if $k \geq m-b$, then $r\left(T_{m}, K_{1, n-1}\right)=m+n-3$.

Theorem 6.3. Let $m, n \in \mathbb{N}, n \geq m \geq 3, m-1 \nmid n-2, n=q(m-2)+a, q \in \mathbb{N}$ and $a \in$ $\{2,3, \ldots, m-3\}$. Let $T_{m} \neq K_{1, m-1}$ be a tree on $m$ vertices. If $n \geq(a-3)(m-1)+3$, then $r\left(T_{m}, K_{1, n-1}\right)=m+n-3$.

Proof. Since $q(m-2)=n-a \geq(a-3)(m-2), q \geq a-3$. Set $G=(a-2) K_{m-1} \cup$ $(q-(a-3)) K_{m-2}$. Then

$$
|V(G)|=(a-2)(m-1)+(q-(a-3))(m-2)=m+n-4 \quad \text { and } \quad \Delta(\bar{G}) \leq n-2 .
$$

Clearly, $T_{m}$ is not a subgraph of $G$ and $K_{1, n-1}$ is not a subgraph of $\bar{G}$. Thus $r\left(T_{m}, K_{1, n-1}\right)>|V(G)|=m+n-4$. By Proposition 6.2, $r\left(T_{m}, K_{1, n-1}\right) \leq m+n-3$. So $r\left(T_{m}, K_{1, n-1}\right)=m+n-3$. This proves the theorem.

Theorem 6.4. Let $m, n \in \mathbb{N}$ with $n>m \geq 5$ and $m-1 \nmid n-2$. Then $r\left(T_{m}^{*}, K_{1, n-1}\right)=$ $m+n-3$ or $m+n-4$. Moreover, if $m+n-4=(m-1) x+(m-2) y+2(m-3) z$ for some nonnegative integers $x, y$ and $z$, then $r\left(T_{m}^{*}, K_{1, n-1}\right)=m+n-3$.

Proof. By Proposition 6.2, $r\left(T_{m}^{*}, K_{1, n-1}\right) \leq m+n-3$. By Lemma 2.3, $r\left(T_{m}^{*}, K_{1, n-1}\right) \geq$ $m+n-4$. If $m+n-4=(m-1) x+(m-2) y+2(m-3) z$ for some nonnegative integers $x, y$ and $z$, setting $G=x K_{m-1} \cup y K_{m-2} \cup z K_{m-3, m-3}$ we find that $\Delta(\bar{G}) \leq n-2$. Clearly, $G$ does not contain any copies of $T_{m}^{*}$, and $\bar{G}$ does not contain any copies of $K_{1, n-1}$. Thus, $r\left(T_{m}^{*}, K_{1, n-1}\right)>|V(G)|=m+n-4$ and so $r\left(T_{m}^{*}, K_{1, n-1}\right)=m+n-3$. This proves the theorem.

\section{References}

[1] S. A. Burr, 'Generalized Ramsey theory for graphs-a survey', in: Graphs and Combinatorics, Lecture Notes in Mathematics, 406 (eds. R.A. Bari and F. Harary) (Springer, Berlin-New York, 1974), pp. 52-75.

[2] S. A. Burr and J. A. Roberts, 'On Ramsey numbers for stars', Util. Math. 4 (1973), 217-220.

[3] P. Erdős and T. Gallai, 'On maximal paths and circuits in graphs', Acta Math. Acad. Sci. Hungar. 10 (1959), 337-356.

[4] G. H. Fan and L. L. Sun, 'The Erdôs-Sós conjecture for spiders', Discrete Math. 307 (2007), 3055-3062.

[5] R. J. Faudree and R. H. Schelp, 'Path Ramsey numbers in multicolorings', J. Combin. Theory Ser. B 19 (1975), 150-160.

[6] Y. B. Guo and L. Volkmann, 'Tree-Ramsey numbers', Aust. J. Combin. 11 (1995), $169-175$. 
[7] L. K. Hua, Introduction to Number Theory (Springer, Berlin, 1982).

[8] S. P. Radziszowski, 'Small Ramsey numbers', Dynamic Surveys of Electronic J. Combinatorics (2011), DS1.13, 84 pp.

[9] A. F. Sidorenko, 'Asymptotic solution for a new class of forbidden $r$-graphs', Combinatorica 9 (1989), 207-215.

[10] Z. H. Sun and L. L. Wang, 'Turán's problem for trees', J. Combin. Number Theory 3 (2011), $51-69$.

[11] M. Woźniak, 'On the Erdôs-Sós conjecture’, J. Graph Theory 21 (1996), 229-234.

ZHI-HONG SUN, School of Mathematical Sciences, Huaiyin Normal University, Huaian, Jiangsu 223001, PR China

e-mail: zhihongsun@yahoo.com 